\title{
Factores asociados a fractura de espina tibial en el hospital Ramiro Prialé Prialé 2015 - 2017
}

\section{Factors associated with tibial spine fracture in Ramiro Prialé Prialé hospital 2015 - 2017}

\author{
'Miranda Huancahuari, M.; Ramírez Castro, C.; Toribio Salazar, L. \\ Facultad de Medicina Humana / Universidad Nacional del Centro del Perú \\ Email:mmiranda@uncp.edu.pe
}

\section{Resumen}

Las fracturas de diáfisis tibiales cerradas son las fracturas de huesos largos más comunes, siendo a su vez las fracturas de espina tibial (FET) que conllevan a inestabilidad y artrosis en la rodilla si no es tratada adecuada y oportunamente. El estudio fue observacional descriptivo con una población de 33 pacientes con fractura de espina tibial. De acuerdo con la clasificación de Meyers y McKeever, el tipo I donde no existe desplazamiento y sólo el labio anterior de la espina están ligeramente elevado, sin exposición, fue el tipo de fractura más frecuente.

Se obtuvo que el hueso tibial izquierdo fue ligeramente más frecuente $(51,5 \%)$ respecto al del lado derecho; el mecanismo de lesión más frecuente fue la hiperextensión de la rodilla $(60,6 \%)$, las causas más frecuentes de la lesión fueron accidente tránsito y la lesión deportiva (ambas representaron el 27,3\%). El 78,8\% de las fracturas no fueron expuestas y de acuerdo con la clasificación de Meyers y McKeever, el tipo I fue el tipo de fractura más frecuente $(57,6 \%)$. Dentro de las afecciones que suelen acompañar a la fractura de espina tibial, sólo un $15 \%$ presentó afección meniscal y; sólo un $12 \%$ presentó afección al platillo tibial.

Palabras Clave: fracturas, diáfisis tibial, espina tibial, platillo tibial, huesos largos

\begin{abstract}
Closed tibial shaft fractures are the most common long bone fractures, being in turn tibial spine fractures (TEF) that lead to instability and osteoarthritis in the knee if not dealt in a proper and timely manner. The study was descriptive observational with a population of 33 patients with tibial spine fracture. According to the Meyers and McKeever classification, type I where there is no displacement and only the anterior lip of the spine is slightly elevated, without exposure, was the most frequent type of fracture.

It was found that the left tibial bone was slightly more frequent $(51,5 \%)$ compared to the right side; the most frequent injury mechanism was hyperextension of the knee $(60,6 \%)$, the most frequent causes of it were traffic accident and sports injury (both represented $27,3 \%) .78,8 \%$ of the fractures were not exposed and according to the Meyers and McKeever classification, type I was the most frequent type of fracture $(57,6 \%)$. Among the conditions that usually accompany tibial spine fracture, only $15 \%$ presented meniscal involvement, and only $12 \%$ presented tibial plateau involvement.
\end{abstract}

Keywords: fractures, tibial diaphysis, tibial spine, tibial plateau, long bones 


\section{Introducción}

Dentro de las fracturas de miembro inferior, las fracturas de diáfisis tibiales cerradas son las fracturas de huesos largos más comunes, siendo a su vez las fracturas de espina tibial (FET) una entidad relativamente infrecuente (Andersen et al., 1993); sin embargo, tiene relevancia debido a que este tipo de fracturas conlleva a inestabilidad y artrosis en la rodilla, si no es tratada adecuada y oportunamente. Este tipo de fracturas está determinado como la solución de continuidad de la espina tibial, por encima de la metáfisis, sitio que es traccionado por la inserción del ligamento cruzado anterior en su inserción distal.

De acuerdo con lo reportado en un inicio por la literatura, la población infantil representa el mayor número de casos de FET (Bakalim y Wilppula, 1974; Baxter y Wiley, 1988); sin embargo, ahora se sabe que su prevalencia es muy parecida en la población adulta (Berg, 1993). Su mayor incidencia se presenta en los adultos jóvenes posterior a un traumatismo de rodilla, ya sea por un accidente de tránsito, caídas o lesiones deportivas, las cuales, al generar una importante fuerza sobre el ligamento cruzado producen, como consecuencia, una fractura por avulsión de la espina tibial.

Además, se ha reportado que en general, las causas principales de este tipo de fracturas, están compuestas por los traumatismos deportivos donde el mecanismo está dado por una hiperextensión forzada de rodilla con rotación tibial con respecto al fémur; seguida de la causa menciona anteriormente, encontramos los accidentes de tránsito y traumatismo directo sobre la tibia.

Las fracturas de espina tibial se clasifican, según Meyers y McKeever - Zacaricznyj, de acuerdo a la magnitud del desplazamiento de los fragmentos examinados radiológicamente y se respaldan en la clasificación propuesta inicialmente en cuatro tipos (Bakalim y Wilppula, 1974):

- Fractura tipo I: solo el labio anterior de la espina está ligeramente elevado

- Fractura tipo II: el tercio o la mitad anterior del fragmento esta elevado conservando la inserción del borde posterior.

- Fractura tipo IIIa: todo el fragmento esta desplazada sin contacto con la tibia

- Fractura tipo IIIb: la eminencia esta desplazada y rotada

- Fractura tipo IV: se agrega conminación del fragmento desprendido, pudo evidenciarse inestabilidad de la rodilla; además, esta lesión se acompaña algunas veces de lesiones meniscales y ligamentarias asociadas.

Los protocolos actuales proponen inmovilización no quirúrgica del miembro inferior en las fracturas no desplazadas, se procede a inmovilizar la articulación en hiperextensión por un periodo aproximado de 6 semanas; además, de efectuar radiografías de seguimiento cada 2 semanas para identificar cualquier desplazamiento, así como al momento de la extracción del yeso, con el objetivo de evidenciar una cicatrización ósea adecuada (Burstein et al., 1988).

Respecto a la inmovilización quirúrgica, la técnica de fijación más común es la reducción por vía artroscópica con clavos cruzados, donde uno de los clavos se inserta lateral y el otro medial, canulados; asimismo, se pueden emplear supersuturas y tornillos canulados (Fabbriciani y Delcogliano, 1980). Este método da una estabilidad satisfactoria a la fractura; pero, existe también el riesgo de lesionar el cartílago, así como producir rigidez de la rodilla. También, existen complicaciones relacionadas con la fractura de espina tibial como son la inestabilidad de rodilla con ruptura de ligamento cruzado anterior, lesiones meniscales, rigidez de rodilla, entre otras, que pueden agravarse si no se realiza una rehabilitación adecuada (Girgis et al., 1975; Gronkvist y Hirsh, 1992)

Por lo mencionado anteriormente, es necesario e importante que se realicen estudios que permitan conocer la situación actual de las FET, así como determinar sus factores de riesgo, ya que al determinar los mismos, se podrían implementar programas de prevención, abordar de manera óptima a los pacientes, brindándo el tratamiento más adecuado y reduciendo, así, procedimientos innecesarios, estancia hospitalaria y complicaciones.

\section{Métodos y materiales}

\section{Población y muestra}

Se realizó un estudio descriptivo, transversal, observacional. La población, estuvo instituida por todos los pacientes con diagnóstico de fractura de espina tibial confirmada por exámenes auxiliares, tales como radiografía y/o tomografía, ingresados en el Servicio de Traumatología y Ortopedia del Hospital Nacional Ramiro Prialé Prialé - Huancayo, durante enero del 2015 hasta diciembre del 2017.

El cálculo de la muestra, se realizó mediante el programa EPIDAT 4.2, usando datos de prevalencia (0,8 \%) de fractura de espina tibial confirmada por exámenes auxiliares tales como radiografía y/o tomografía del HNRPP del año 2016, con un valor de $\mathrm{p}=0,05$, obteniendo 33 pacientes como cantidad mínima.

Criterios de inclusión. Pacientes con fractura de espina tibial confirmada por radiografía y/o tomografía.

Criterios de exclusión. Pacientes con fractura de espina tibial confirmada por radiografía y/o tomografía que tengan otro tipo de comorbilidades, pacientes politraumatizados y pacientes con inestabilidad hemodinámica.

\section{Técnica de recolección y procesamiento de datos}

Previos criterios de selección, se recolectó datos de las historias clínicas y se rellenó una ficha de recolección 
de datos, donde consignaban todas las variables a estudiar de los pacientes que tuvieron una fractura de espina tibial, reportados por el Servicio de Traumatología y Ortopedia del Hospital Nacional Ramiro Prialé Prialé.

Los datos de la ficha de recolección, se ordenaron en Excel y posteriormente la estadística fue obtenida con el programa STATA 12.

\section{Resultados}

Se incluyeron un total de 33 pacientes que presentaron fractura de espina tibial. La mediana de edad fue de 28 años (rango intercuartílico: 24-33 años), la mayoría fueron varones $(66,7 \%)$ y el lugar del cual procedieron la mayoría de los pacientes fue el distrito de Huancayo (27,3\%), seguido del distrito de El Tambo (21,2 \%), como se muestra en la Tabla 1.

Tabla 1

Características demográficas de la población estudiada

\begin{tabular}{ccc}
\hline & Variable & N (\%) \\
\hline Sexo & Edad (años)* & $28(24-33)$ \\
& & \\
Lugar de procedencia & Maculino & $11(33,3 \% 9$ \\
Femenino & \\
Huancayo & $9(27,3 \%)$ \\
El Tambo & $7(21,2 \%)$ \\
Concepción & $5(15,2 \%)$ \\
Chilca & $4(12,1 \%)$ \\
Pilcomayo & $3(9,0 \%)$ \\
Otros & $5(15,2 \%)$ \\
\hline
\end{tabular}

* Mediana y rangos intercuantílicos.

Respecto a las características de la fractura de espina tibial, el hueso tibial izquierdo fue ligeramente más frecuente $(51,5 \%)$ respecto al del lado derecho; el mecanismo de lesión más frecuente fue la hiperextensión de la rodilla $(60,6 \%)$.

Las causas más frecuentes de la lesión fueron accidente tránsito y la lesión deportiva (ambas representaron el $27,3 \%$ ). El 78,8 \% de las fracturas no fueron expuestas y de acuerdo con la clasificación de Meyers y McKeever, el tipo I donde no existe desplazamiento y sólo el labio anterior de la espina está ligeramente elevado, fue el tipo de fractura más frecuente $(57,6 \%)$.

Dentro de las afecciones que suelen acompañar a la fractura de espina tibial; solo un $15 \%$, presentó afección meniscal y; solo un $12 \%$, presentó afección al platillo tibial (Tabla 2).
Tabla 2

Características clínicas de la fractura de espina tibial

\begin{tabular}{cc}
\hline Variable & N (\%) \\
\hline Miembro inferior fracturado & \\
Derecho & $16(48,5 \%)$ \\
Izquierdo & $17(51,5 \%)$ \\
Mecanismo de lesión & \\
Hiperextensión & $20(60,6 \%)$ \\
Avulsión & $10(30,3 \%)$ \\
Torsión & $3(9,1 \%)$ \\
Causa de lesión & \\
Accidente de tránsito & $9(27,3 \%)$ \\
Lesión deportiva & $9(27,3 \%)$ \\
Caída & $8(24,2 \%)$ \\
Caída de bicicleta & $7(21,2 \%)$
\end{tabular}

\section{Fractura expuesta}

$\begin{array}{cc}\text { Si } & 7(21,2 \%) \\ \text { No } & 26(78,8 \%)\end{array}$

\section{Clasificación según Meyers y McKeever}

$\begin{array}{cc}\text { Tipo I } & 19(57,6 \%) \\ \text { Tipo II } & 6(18,2 \%) \\ \text { Tipo III } & 4(12,1 \%) \\ \text { Tipo IV } & 4(12,1 \%)\end{array}$

Afecta a menisco

$$
\begin{array}{cc}
\text { Si } & 5(15,1 \%) \\
\text { No } & 28(84,6 \%)
\end{array}
$$

Afecta a platillo tibial

\begin{tabular}{cc} 
Si & $4(12,1 \%)$ \\
No & $29(87,9 \%)$ \\
\hline
\end{tabular}

\section{Discusión}

Después de analizar los resultados, se observó que el sexo masculino presentó mayor prevalencia de fracturas de espina tibial $(66,7 \%)$. Esto concuerda con dos estudios; uno realizado en España, en el cual los varones también fueron los que presentaron más esta fractura, tanto en los niños (54,5\%) como en los adultos (78 \%); además, en este mismo estudio se muestra que la edad promedio en los niños fue de 12 años y de los adultos fue de 38 años, lo que se asemeja a nuestra población en la cual la edad promedio fue de 28 años.

El otro estudio fue realizado en una población joven, en el cual los varones representaron más de la mitad de casos (69\%), cifra que es muy parecida la obtenida por el presente estudio. 
En la presente investigación, se observa que el miembro inferior más afectado fue el izquierdo (57,5\%), al igual que en el estudio realizado en España en el que en los niños y adultos, el lado más afectado fue, también, el izquierdo.

El mecanismo de lesión más frecuente de fracturas fue la hiperextensión y la causa más frecuente fue un accidente de tránsito $(27,3 \%)$ o una lesión deportiva $(27,3 \%)$. En un estudio español, se mostró que en niños y adultos, el accidente de tránsito fue la causa más prevalente (36,3\% y 44,5\% respectivamente). En otro estudio, se evidenció que de los 105 pacientes con diagnóstico de FET, la causa más frecuente de la lesión fueron las caídas (30,47\%).

Según la clasificación de Meyer, el tipo de fractura más frecuente encontrado fue el tipo I; este hallazgo difiere con otra investigación, en el cual el tipo más frecuente fue el II. En un estudio se muestra que las lesiones asociadas fueron periarticulares (rotura del menisco, rotura del ligamento lateral, fractura hundimiento del platillo tibial; asi mismo, en otro, se menciona que la afección del menisco es una de las lesiones concomitantes a fractura de espina tibial más frecuente; en la presente investigación, se observó que el $15 \%$, vio afectado el menisco y; el $12 \%$, el platillo tibial.

\section{Conclusiones}

- Respecto a las características de la fractura de espina tibial, el hueso tibial izquierdo fue ligeramente más frecuente; el mecanismo de lesión más frecuente fue la hiperextensión de la rodilla; dentro de las causas más frecuentes de lesión, están los accidentes de tránsito y la lesión deportiva.

- De acuerdo con la clasificación de Meyers y McKeever, el tipo I donde no existe desplazamiento y sólo el labio anterior de la espina está ligeramente elevado, fue el tipo de fractura más frecuente, las fracturas no expuestas fueron las más frecuentes. Dentro de las afecciones que suelen acompañar a la fractura de espina tibial, sólo un pequeño porcentaje presentó afección meniscal y de platillo tibial.

- Además, se obtuvo que el hueso tibial izquierdo fue ligeramente más frecuente $(51,5 \%)$ respecto al lado derecho; el mecanismo de lesión más fre- cuente fue la hiperextensión de la rodilla $(60,6 \%)$, las causas más frecuentes de la lesión fueron accidente tránsito y la lesión deportiva (ambas representaron el 27,3\%).

- El 78,8 \% de las fracturas no fueron expuestas y, de acuerdo con la clasificación de Meyers y McKeever, el tipo I donde no existe desplazamiento y sólo el labio anterior de la espina está ligeramente elevado, fue el tipo de fractura más frecuente $(57,6 \%)$. Dentro de las afecciones que suelen acompañar a la fractura de espina tibial, solo un $15 \%$ presentó afección meniscal y, solo un $12 \%$ presentó afección al platillo tibial.

\section{Referencias bibliográficas}

Andersen, J. W. \& Meudhal, S. (1993). Bilateral fractures of the tibia spine. Acta Orthop Belg; 59 (1): 394-397.

Bakalim, G. \& Wilppula, E. (1974). Closed treatment of fractures of the tibial spine. Injury; 5 (1). 210.

Baxter, M. \& Wiley, J. (1988). Fractures of the tibial spine in children. JBone J Surg; 70-B (2)

Berg, E. E. (1993). Comminuted tibial eminence anterior cruciate ligament avulsión fractures: failure of arthroscopic treatment. Arthroscopy; 9 (4) 446 $-450$

Burstein, D. B.; Viola, A. \& Fulkerson, J. P. (1988). Entrapment of the medial meniscus in a fracture of the tibial eminence arthroscopy; 4: $47-50$.

Fabbriciani, D. \& Delcogliano, A. (1980). Recent fractures of the tibial spine. Diagnostic and therapeutic criteria. Arch Putti Chir Organi Mov; 30231 -247

Girgis, F. B.; Marshall, J. L. \& Al Monajem, A. R. (1975). The cruciate ligaments of the knee joint: Anatomical functioinal and experimental analysis. Clin Orhop; $106: 210$

Gronkvist, H. \& Hirsh, G. (1992). Fracture of the anterior tibial spine in childrens. J Bone Jt Surg; 74b (6): 848 